\title{
"El corazón más grande que la caja": Un caso de Cor Pulmonale
}

\author{
Pablo Franquelo Morales ${ }^{a}$, Achille Reagens ${ }^{a}$, Félix González Martínez ${ }^{a}$, David García Mateos ${ }^{a}$
}

a Servicio de Urgencias. Hospital Virgen de la Luz, Cuenca.

\section{Correspondencia:}

Pablo Franquelo Morales. Servicio de Urgencias, Hospital Virgen de la Luz, $\mathrm{C} /$ Hermandad de Donantes de Sangre $n^{\circ} 2$,

16002-Cuenca (España).

E-mail: pfranquelo@yahoo.es.

Recibido el 21 de marzo de 2009.

Aceptado para su publicación el 17 de mayo de 2009.

\section{RESUMEN}

El Cor Pulmonale (CP) se produce como resultado de un aumento de la presión en los pulmones, lo que origina un incremento en el tamaño del ventrículo derecho (VD) por sobrecarga. Aproximadamente un $20-30 \%$ de los ingresos hospitalarios por insuficiencia cardiaca congestiva se debe a insuficiencia ventricular derecha asociada a CP. La causa más común es la EPOC (enfermedad pulmonar obstructiva crónica). La clínica, el electrocardiograma y la radiografía de tórax son inespecíficos. El método diagnóstico no invasivo de elección es el ecocardiograma-doppler, pero el cateterismo cardiaco es la prueba gold estándar. El tratamiento se basa en diuréticos, vasodilatadores y oxígeno a largo plazo.

Palabras clave. Enfermedad Cardiopulmonar. Enfermedad Pulmonar Obstructiva Crónica. Hipertensión Pulmonar

\section{ABSTRACT}

"The heart bigger than the box": a case of Cor Pulmonale

Cor pulmonale (CP) occurs when there is increased pressure in the lungs, causing enlargement of the right ventricle (VD) due to pressure overload. Approximately $20-30 \%$ of hospital admissions for congestive heart failure are due to right ventricular failure associated with $\mathrm{CP}$. The most common cause is COPD (chronic obstructive pulmonary disease). The clinical, electrocardiogram, and chest $\mathrm{X}$-ray examinations are non-specific. Doppler echocardiogram is the non-invasive diagnostic method of choice is, however cardiac catheterization is the gold standard. Treatment is based on diuretics, vasodilators and long-term oxygen.

Key words. Pulmonary Heart Disease. Pulmonary Disease, Chronic Obstructive. Hypertension, Pulmonary.

\section{INTRODUCCIÓN}

Hace unos días durante el transcurso de una guardia, uno de los residentes, de origen haitiano, comentó que su paciente decía tener "el corazón más grande que la caja".Tras proceder a explicar el significado de la expresión, nos pareció interesante profundizar en el concepto de Cor Pulmonale (CP).

\section{OBSERVACIONES CLÍNICAS}

Se trata de un varón de 77 años con antecedentes de insuficiencia renal crónica, hipertensión arterial de larga evolución y EPOC, que consultó por episodio de empeoramiento de su disnea habitual en los últimos días en el contexto de un cuadro catarral. Había sido fumador de 30 paquetes/año durante 40 años, pero hacía más de una década que había abandonado este hábito. Presentaba episodios frecuentes de descompensación de insuficiencia cardiaca derecha crónica que obligaban a su ingreso hospitalario. El tratamiento habitual consistía en telmisartán, furosemida, amlodipino y doxazosina, así como en la administración de oxígeno domiciliario durante más de 16 horas al día.

En la exploración física se mostró consciente y orientado, con aspecto abotargado e ingurgitación yugular a $90^{\circ}$. Registramos cifras de TA de 120/70 mm/Hg, Ta $36 \mathrm{C}^{\circ}$, Saturación de 02 al 35\% (10 lpm) de 87\%, con presencia de flapping y disminución del murmullo vesicular mas espiración prolongada en los campos pulmonares. En 
la auscultación cardiaca se apreció un soplo sistólico en el foco mitral, con presencia de hepatomegalia de 2-3 traveses, así como llamativos edemas y signos de insuficiencia venosa crónica en las extremidades inferiores.

En las pruebas complementarias se objetivó una leucocitosis de $13.700 \mathrm{mil} / \mathrm{mmc}$, Hb de 13,1 g/dl y dímero D de $2.400 \mathrm{~g} / \mathrm{L}$, así como valores de creatinina de $3,1 \mathrm{mg} / \mathrm{dl}$, urea de $170 \mathrm{mg} / \mathrm{dL}$ y PCR de $6,8 \mathrm{mg} / \mathrm{L}$. En la orina se hallaron 10 hematíes por campo, y una gasometría arterial basal con pO2 de $53 \mathrm{~mm} / \mathrm{Hg}, \mathrm{pCO} 2$ de $56 \mathrm{~mm} / \mathrm{Hg}$ y $\mathrm{pH}$ de 7.37 .

El electrocardiograma (ECG) reveló un ritmo sinusal con presencia de $\mathrm{p}$ "pulmonale" en la cara inferior e hipertrofia del ventrículo derecho. La radiografía de tórax mostró signos de hipertensión arterial pulmonar (HTP), cardiomegalia global, y aumento difuso de la trama bronco-vascular (figura 1).

Fue tratado con broncodilatadores, antibióticos y diuréticos, mejorando gradualmente la situación respiratoria y los signos de insuficiencia cardiaca, emitiéndose el alta hospitalaria con diagnóstico final de insuficiencia cardiaca crónica de predominio derecho, CP crónico, EPOC reagudizado por infección respiratoria, obesidad, insuficiencia respiratoria hipercápnica, insuficiencia renal crónica e HTA.

\section{COMENTARIOS}

El CP se produce como resultado de un aumento de la presión en los pulmones, lo que origina un incremento en el tamaño del ventrículo derecho (VD) por sobrecarga $^{1,2}$. Esto se debe a enfermedades que afectan al pulmón, el tórax o a la circulación pulmonar. Teniendo en cuenta que el VD desplaza hacia los pulmones la sangre desoxigenada que proviene de la circulación sistémica con la finalidad de oxigenarse de nuevo mediante la respiración, al existir algún trastorno en la circulación pulmonar, este proceso se ve afectado, lo que genera una $\mathrm{HTP}^{3,4}$, que repercute en el corazón, obligándole a incrementar el trabajo cardiaco para poder mantener la circulación sanguínea pulmonar y vencer la resistencia que opone dicho aumento de presión ${ }^{1,3,5}$. Aproximadamente un $20-30 \%$ de los ingresos hospitalarios por insuficiencia cardiaca congestiva se debe a insuficiencia ventricular derecha asociada a CP, con un predominio en el sexo masculino ${ }^{1}$. Las causas más comunes son los trastornos pulmonares crónicos, sobre todo la EPOC, presente en más del 50\%6 (tabla1).

El diagnóstico de CP es complicado debido a que los síntomas pueden estar en relación con la propia enfermedad pulmonar, y además la exploración física, con datos orientativos como soplos de regurgitación tricúspidea, ingurgitación yugular o aumento del $2^{\circ}$

\begin{tabular}{|c|c|c|}
\hline Enfermedades pulmonares obstructivas & Enfermedades pulmonares restrictivas & Insuficiencia respiratoria de origen central \\
\hline $\begin{array}{l}\text { EPOC* (Bronquitis crónica, } \\
\text { enfisema o su asociación) }\end{array}$ & Cifoescoliosis & $\begin{array}{l}\text { Síndrome obesidad- } \\
\text { hipoventilación }\end{array}$ \\
\hline Fibrosis quística & Neumoconiosis & SAOS $^{*}$ \\
\hline Bronquiectasias & Fibrosis pulmonar intersticial & \\
\hline
\end{tabular}

Tabla1. Causas más frecuentes de Cor Pulmonale e Hipertensión Pulmonar. *EPOC: Enfermedad pulmonar obstructiva crónica. **SAOS: Síndrome de apnea obstructiva del sueño.

Figura 1. Radiografía de tórax PA con redistribución vascular y aumento del índice cardiotorácico.

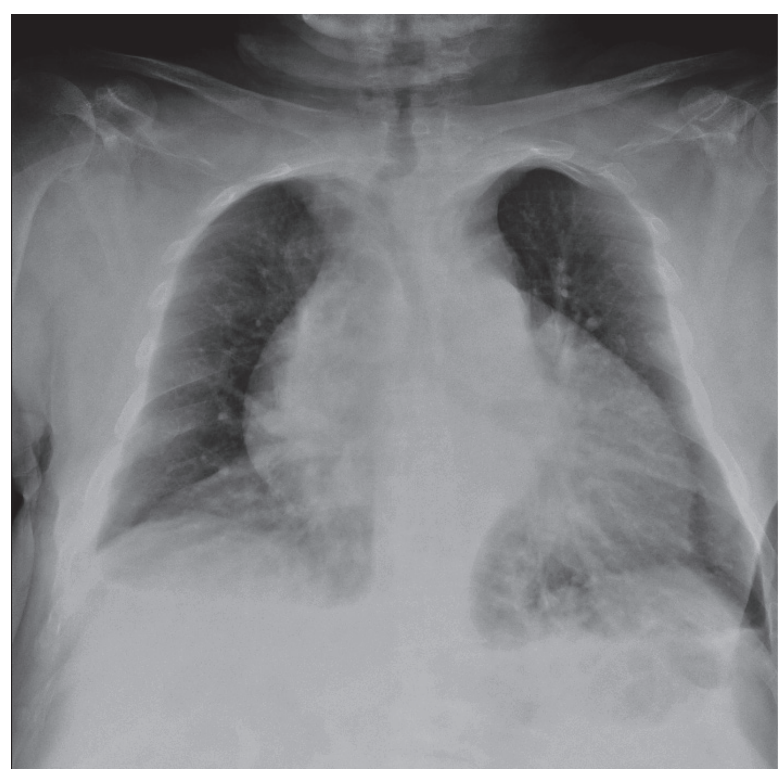


tono, puede estar enmascarada en los pacientes con EPOC1. Por otro lado, la clínica, consistente en disnea o astenia, así como el electrocardiograma, con hipertrofia ventricular derecha, y la radiografía de tórax, que muestra una dilatación de los hilios pulmonares a expensas de las ramas arteriales pulmonares y un ventrículo derecho dilatado ${ }^{7}$, son bastante inespecíficas. El método diagnóstico no invasivo de elección es el ecocardiograma-doppler ${ }^{8}$, y la resonancia magnética (RM) puede resultar muy útil para la valoración anatómica del ventrículo derecho ${ }^{1}$. No obstante, la toma de presiones en cavidades derechas y en la arteria pulmonar mediante cateterismo cardiaco constituye la prueba gold estándar, pero al ser una técnica invasiva su uso se limita a indicaciones muy concretas.

El tratamiento del CP crónico se basa en el manejo de la insuficiencia cardiaca derecha con diuréticos, para disminuir los edemas periféricos, así como la reducción de la HTP mediante vasodilatadores tipo antagonistas del calcio y oxígeno a largo plazo. En determinadas situaciones se usa digoxina, si se asocia fibrilación auricular o disfunción ventricular izquierda con clínica de insuficiencia cardíaca. Los vasodilatadores pulmonares selectivos como el óxido nítrico inhalado, las prostaciclinas, el sildenafilo y los antagonistas del receptor de la endotelina todavía se encuentran en fase de validación clínica.

\section{BIBLIOGRAFÍA}

1. Gómez-Doblas JJ, Velasco JL, Alarcón López P, Montiel Trujillo A. Cor pulmonale. Etiopatogenia. Proceso diagnóstico.Criterios pronósticos. Tratamiento. Medicine 2005; 09:2873-8.

2. Rodríguez Fernández JÁ, Garrido Bravo IP, Hermida Álvarez LF, Castro Beiras A. Insuficiencia cardiaca crónica. Etiología, manifestaciones clínicas y criterios diagnósticos. Medicine 2005; 09:2300-7.

3. Kessler R, Faller M, Weitzenblum E, Chaouat A, Agkut A, Ducolone $\mathrm{A}$, et al. Natural History of pulmonary hypertension in a series of 131 patients with chronic obstructive lung disease. Am J Respir Crit Care Med 2001; 164:219-24.

4. Naeije R. Pulmonary Hypertension and right Heart Failure in Chronic Obstructive Pulmonary Disease. Proc Am Thorac Soc 2005; 2:20--2.

5. Weitzenblum E. Chronic cor pulmonale. Heart 2003; 89:225-30.

6. Barbera JA, Peinado V, Santos S. Pulmonary hypertension in chronic obstructive pulmonary disease. Eur Respir J 2003; 21:892-905.

7. Gerber BL, Melin JA, Vanoverschelde JL. Imaging the heart in patients with cardiac failure. En: Michael Peters A, editor. Nuclear Medicine in Radiological Diagnosis. United Kingdom: Martin Dunitz; 2003. p. 363-77.

8. Matsuyama W, Ohkubo R, Michizono K, Abe M, Nakamura Y, Kawabata M, et al. Usefulness of transcutaneous Doppler jugular venous echo to predict pulmonary hypertension in COPD patients. Eur Respir J 2001; 17:1128-31.Síndrome obesidad-hipoventilación. 